C-A/AP/\#421 Jan. 2011

\title{
Multipole Error Data Analysis for RHIC Low-Energy Operations
}

\author{
C. Montag
}

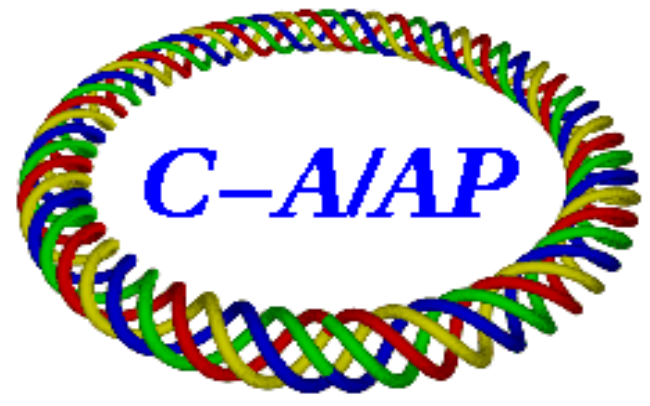

Collider-Accelerator Department Brookhaven National Laboratory Upton, NY 11973 


\title{
Multipole error data analysis for RHIC low-energy operations
}

\author{
Christoph Montag
}

January 14, 2011

\section{Run-10 experience at low energies}

In FY10, RHIC operated with gold beams at three different energies below its regular injection energy, namely at $\sqrt{s}=5 \mathrm{GeV}, 7.7 \mathrm{GeV}$, and $11.5 \mathrm{GeV}$. While beam lifetimes of about 10 minutes were achieved at $11.5 \mathrm{GeV}$ and $7.7 \mathrm{GeV}$, leading to stable collider operation, beam lifetimes during tests at $\sqrt{s}=5 \mathrm{GeV}$ were only of the order of several seconds. Though space charge effects are a major concern in low energy operations due to the $1 / \gamma^{2}$ scaling of the space charge force, these can effectively be ruled out as the main cause of this abysmal performance because of the tiny bunch intensities of approximately $10^{8} \mathrm{Au} / \mathrm{bunch}$, compared to $10^{9} \mathrm{Au} /$ bunch at the two higher energies.

During these $5 \mathrm{GeV}$ tests, large betatron tune spreads were observed, but the extremely short beam lifetime prevented chromaticity measurements. Instead, chromaticities were scanned over a range of 40 units, but to no effect. It was therefore suspected at that time that the large tune spread was caused by higherorder multipole errors in the RHIC magnets.

\section{Measured multipole errors}

In preparation for the low-energy run in FY10, dedicated multipole measurements were performed on one spare dipole and one spare quadrupole magnet each. The magnets were cycled through a hysteresis cycle with a maximum field corresponding to $100 \mathrm{GeV}$ proton operations. At various field values along the hysteresis cycle, the main field component as well as the two lowest allowed multipole errors (sextupoles and decapoles for dipoles, dodecapoles and 20-poles for quadrupoles) were measured with a rotating coil. The multipole components thus obtained are depicted in Figure 1.

For an easier comparison of the various multipole components at different energies, the sextupole, 10-, 12-, and 20-pole components for $\sqrt{s}=5 \mathrm{GeV}$, $\sqrt{s}=7.7 \mathrm{GeV}$, regular injection energy, and $100 \mathrm{GeV}$ proton beam energy are listed in Table 1. 

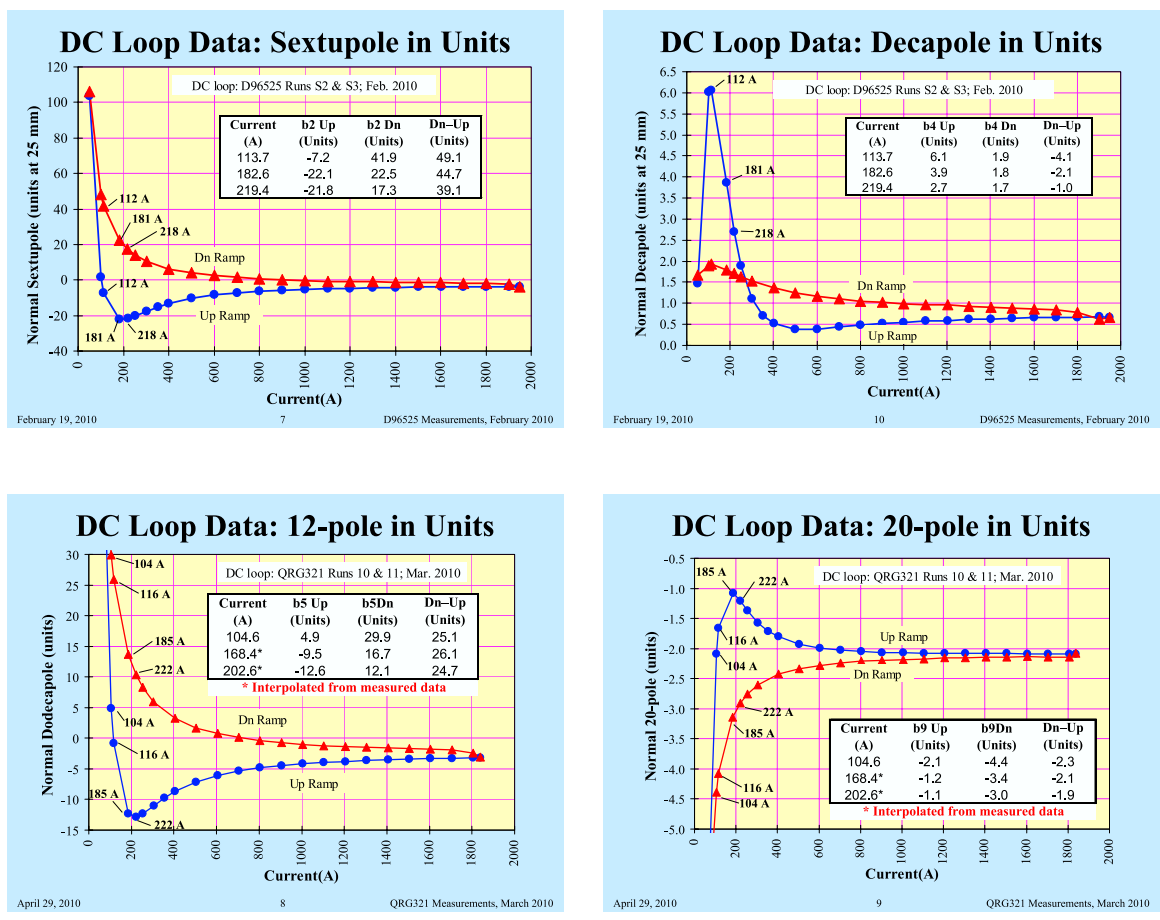

Figure 1: Measured multipole components in one spare arc dipole and one spare arc quadrupole magnet each.

Table 1: Comparison of measured multipoles at four different energies.

\begin{tabular}{|l|c|c|c|c|}
\hline \hline & $\sqrt{s}=5 \mathrm{GeV}$ & $\sqrt{s}=7.7 \mathrm{GeV}$ & $\begin{array}{c}\text { regular } \\
\text { injection }\end{array}$ & $\begin{array}{c}100 \mathrm{GeV} \\
\text { protons }\end{array}$ \\
\hline sextupole & -7.2 & -22.1 & -10 & -3 \\
10-pole & 6.1 & 3.9 & 0.4 & 0.7 \\
12-pole & 4.9 & -9.5 & -7 & -3 \\
20-pole & -2.1 & -1.2 & -1.9 & -2.1 \\
\hline \hline
\end{tabular}


As already mentioned earlier, beam lifetimes were of the order of 10 minutes at $\sqrt{s}=7.7 \mathrm{GeV}$, and they are significantly longer at regular injection energy and store energy. We can therefore conclude that the multipole components corresponding to these energies are not particularly harmful to the beam lifetime.

Comparing the multipoles for $\sqrt{s}=5 \mathrm{GeV}$ with those at these higher energies, we make the following observations:

- The sextupole component at $\sqrt{s}=5 \mathrm{GeV}$ is comparable to the one at regular injection energy, and a factor three smaller than at $\sqrt{s}=7.7 \mathrm{GeV}$;

- the 10-pole is about 50 percent larger at $\sqrt{s}=5 \mathrm{GeV}$ than at $\sqrt{s}=$ $7.7 \mathrm{GeV}$;

- the magnitude or the 12 -pole at $\sqrt{s}=5 \mathrm{GeV}$ is about half as large as at $\sqrt{s}=7.7 \mathrm{GeV}$, but has the opposite sign than at the other three energies;

- the 20-pole component at $\sqrt{s}=5 \mathrm{GeV}$ is practically the same as at regular injection energy.

Based on these observations, we can therefore conclude that the poor lifetime performance at $\sqrt{s}=5 \mathrm{GeV}$ was most likely not caused by excessive multipole components at this energy.

\section{$3 \quad$ Modeling issues}

In the model, the sextupole component $b_{2}$ as function of energy is calculated by a fit function, based on measured data between regular injection and store energies. This fit function was not modified for low energy operations; instead, the existing fit was extrapolated towards low energies. As indicated in Figure 2, this results in a good prediction of $b_{2}$ at $\sqrt{s}=7.7 \mathrm{GeV}$, while the predicted value for $b_{2}$ at $\sqrt{s}=5 \mathrm{GeV}$ is around -30 units, instead of the correct value of -7.2 units. Taking into account the fact that one unit of $b_{2}$ results in approximately 5 units of chromaticity, we therefore conclude that during the $\sqrt{s}=5 \mathrm{GeV}$ test the chromaticity was off by roughly 100 units.

This huge chromaticity explains the large tune spread observed during the test, and why even the performed chromaticity scan over 40 units was not sufficient to improve the situation. For future runs at $\sqrt{s}=5 \mathrm{GeV}$, the measured $b_{2}$ data have to be included properly in the model. The difficulty here lies in the fact that these measurements were taken on a single spare dipole magnet. Based on this data set, the $b_{2}$ component of all the RHIC dipoles has to be inferred in a meaningful manner to allow chromaticity prediction within an error margin of \pm 10 units.

While there is no information available on triplet quadrupole errors at low energy, these are not expected to dominate the beam dynamics due to the small number of magnets involved and the moderate $\beta$-functions in the triplets during low-energy operations with $\beta^{*}=10 \mathrm{~m}$, unless triplet quadrupoles perform significantly worse at low energy than arc magnets. 


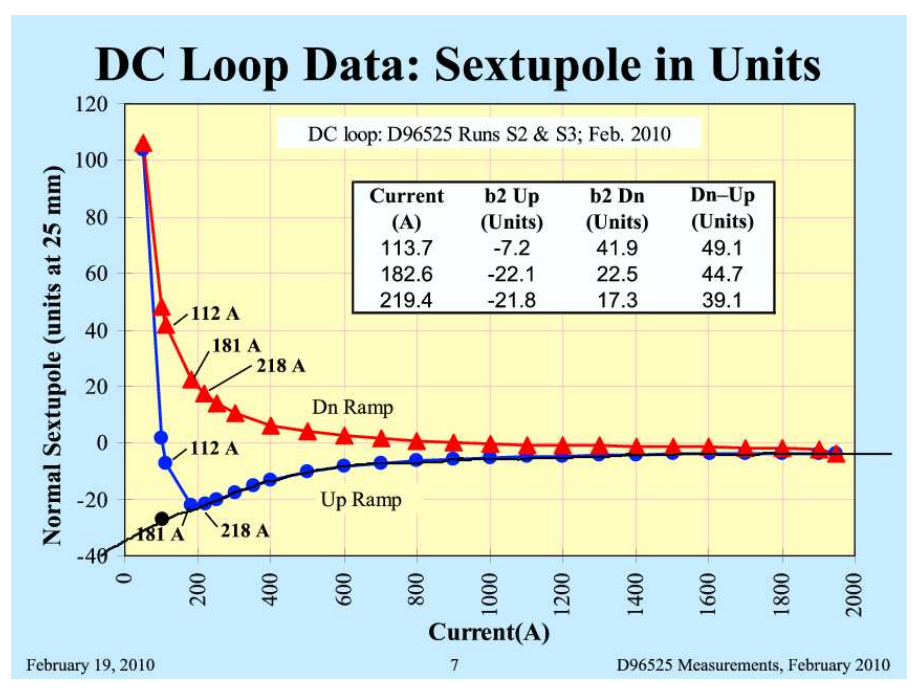

Figure 2: Measured sextupole component $b_{2}$ in the spare dipole. The black line indicates the extrapolation of data between regular injection and store to low energies, as used during the test in FY10. At a magnet current of $113.7 \mathrm{~A}$, which correponds to $\sqrt{s}=5 \mathrm{GeV}$, this extrapolation predicts $b_{2}=-30$ units, while the measured value is $b_{2}=-7.2$ units.

\section{Summary}

During FY10, RHIC performed well at $\sqrt{s}=7.7 \mathrm{GeV}$ and above, while at $\sqrt{s}=$ $5 \mathrm{GeV}$ the beam lifetime was only seconds, even at tiny intensity. This poor performance cannot be explained by measured main magnet multipole errors. Instead, the most likely explanation is a totally wrong main dipole $b_{2}$ component in the model that resulted in a chromaticity discrepancy between machine and model of roughly 100 units. Tracking studies are required to determine whether 50 percent larger 10-pole and opposite sign for 12-pole play any significant role as well, including the "bipolar" sextupole scheme [1] used during operations.

\section{Acknowledgments}

I would like to thank Joanne Beebe-Wang, Guillaume Robert-Demolaize, and Animesh Jain for fruitful discussions.

\section{References}

[1] C. Montag et al., "Mimicking bipolar sextupole power supplies for low-energy oerpations at RHIC", to be published 